\title{
Optimization of the Correcting Gains of Fractional Order Hold Devices for Inter-Sample Output Reconstruction
}

\author{
M. De La Sen and A. Bilbao \\ Instituto de Investigacion y Desarrollo de Procesos, \\ Facultad de Ciencias, Universidad del Pais Vasco, \\ Leioa (Bizkaia). Aptdo. 644 de Bilbao. 48080-Bilbao, Spain
}

\begin{abstract}
The design of Fractional Order Holds (FROH) of correcting gains $\beta \in[-1,1]$ (potentially and possibly including zero-order holds, $\mathrm{ZOH}$ with $\beta=0$, and firelation holds, FROH relating) is discussed related to achieving output deviations being close with respect to its sampled values. A squared error time- integral between the current output and its sampled values is minimized to yield the appropriate correcting gain of the FROH in an analytic way.
\end{abstract}

Key words: Zero, first and fractional-order holds, inter-sample behavior, signal reconstruction

\section{INTRODUCTION}

Continuous Plant: Consider the linear time-invariant single-input single-output plant:

$$
\dot{\mathrm{x}}(\mathrm{t})=\mathrm{Ax}(\mathrm{t})+\mathrm{bu}(\mathrm{t}) ; \mathrm{y}(\mathrm{t})=\mathrm{c}^{\mathrm{T}} \mathrm{x}(\mathrm{t})
$$

where, $\quad \mathrm{x}(\mathrm{t}) \in \mathrm{R}^{\mathrm{n}}, \mathrm{y}(\mathrm{t}) \in \mathrm{R}, \mathrm{u}(\mathrm{t}) \in \mathrm{R}$ are the state vector, and scalar input and output, respectively and $\mathrm{A} \in \mathrm{R}^{\mathrm{n} \times \mathrm{n}} ; \mathrm{b}, \mathrm{c} \in \mathrm{R}^{\mathrm{n}}$. A fractional order -hold generates an input of the form:

$$
\begin{aligned}
& \mathrm{u}(\mathrm{kT}+\tau)=\mathrm{u}_{\mathrm{k}}+\frac{\beta}{\mathrm{T}} \tilde{\mathrm{u}}_{\mathrm{k}-1} ; \quad \beta \in\left[\beta_{1}, \beta_{2}\right] ; \tilde{\mathrm{u}}_{\mathrm{k}-1}=\mathrm{u}_{\mathrm{k}}-\mathrm{u}_{\mathrm{k}-1} ; \\
& \forall \tau \in[0, \mathrm{~T})
\end{aligned}
$$

with $\mathrm{u}_{\mathrm{k}}=\mathrm{u}(\mathrm{kT})$ is the $\mathrm{k}$-th sample of the input with sampling period $\mathrm{T}$. Usually, $\beta_{2}=-\beta_{1}=1$. If $\beta=0$ or $\beta=1$ the $\mathrm{FROH}$ is a $\mathrm{ZOH}$ or at $\mathrm{FOH}$, respectively. Fractionalorder holds have been studied related to the improvement of the stability of the discrete plant zeros which is of interest in problems like poleplacement or model-matching controller synthesis since the reference model might be designed with more relaxed a prior constraint on the need of include prefixed plant unstable zeros ${ }^{[1-4]}$. In this study, we discuss the choice of fractional order holds as a way to improve the deviations of the inter-sample reconstructed output signals compared to their sampled values compared to the use of the standard zero-order and firstorder holds.

Inter-sample State Description: Assume that (1) is discretized with a sampling period $\mathrm{T}$ so that the state evolution (1) becomes for any $\tau \in[0, T)$ :

$$
\begin{aligned}
& \dot{\mathrm{x}}(\mathrm{kT}+\tau)=\mathrm{Ax}(\mathrm{kT}+\tau)+\mathrm{b}\left(\mathrm{u}_{\mathrm{k}}+\beta \mathrm{T}^{-1} \tilde{\mathrm{u}}_{\mathrm{k}-1} \tau\right) \\
& \tau \in[0, \mathrm{~T})
\end{aligned}
$$

What leads to a solution:

$$
\begin{aligned}
& \mathrm{x}(\mathrm{kT}+\tau)=\Phi(\tau) \mathrm{x}_{\mathrm{k}}+\Gamma(\tau) \mathrm{u}_{\mathrm{k}}+\beta \mathrm{T}^{-1} \Gamma^{\prime}(\tau) \tilde{\mathrm{u}}_{\mathrm{k}-1} \\
& \tau \in[0, \mathrm{~T})
\end{aligned}
$$

Where:

$$
\begin{aligned}
& \Phi(\tau)=\mathrm{e}^{\mathrm{A} \tau} ; \quad \Gamma(\tau)=\int_{0}^{\tau} \Phi\left(\tau-\tau^{\prime}\right) \mathrm{bd} \tau^{\prime} \\
& \Gamma^{\prime}(\tau)=\int_{0}^{\tau} \Phi\left(\tau-\tau^{\prime}\right) \mathrm{b} \tau^{\prime} \mathrm{d} \tau^{\prime}
\end{aligned}
$$

for all $\tau \in[0, T)$ and the substitution of (3) into (2) leads to:

$$
\begin{aligned}
& \dot{\mathrm{x}}(\mathrm{kT}+\tau)=\mathrm{A} \Phi(\tau) \mathrm{x}_{\mathrm{k}}+(\mathrm{A} \Gamma(\tau)+\mathrm{b}) \mathrm{u}_{\mathrm{k}} \\
& +\mathrm{T}^{-1} \beta\left(\mathrm{A} \Gamma^{\prime}(\tau)+\mathrm{b} \tau\right) \tilde{\mathrm{u}}_{\mathrm{k}-1}
\end{aligned}
$$

Taking time-derivatives in (2) with respect to time in $(0, \mathrm{~T})$ via $(3)$ yields:

$$
\begin{aligned}
& \ddot{\mathrm{x}}(\mathrm{kT}+\tau)=\mathrm{A}^{2} \Phi(\tau) \mathrm{x}_{\mathrm{k}}+\mathrm{A}(\mathrm{A} \Gamma(\tau)+\mathrm{b}) \mathrm{u}_{\mathrm{k}} \\
& +\mathrm{T}^{-1} \beta\left[\mathrm{A}\left(\mathrm{A}^{\prime}(\tau)+\mathrm{b} \tau\right)+\mathrm{b}\right] \tilde{\mathrm{u}}_{\mathrm{k}-1}
\end{aligned}
$$

Approximate Output Description of the Intersample Behavior: For $\tau \in(0,(1-\rho) \mathrm{T})$, first and second-order Taylor series approximations about , $\mathrm{t}=(\mathrm{k}+\rho) \mathrm{T} \in(\mathrm{kT},(\mathrm{k}+1) \mathrm{T})$ using the output equation (1) and (5)-(6) yield: 


$$
\begin{aligned}
\mathrm{y}((\mathrm{k}+\rho) \mathrm{T}+\tau) \cong y((k+\rho) \mathrm{T})+\dot{y}((\mathrm{k}+\rho) \mathrm{T}) \tau+\alpha \ddot{y}((\mathrm{k}+\rho) \mathrm{T}) \frac{\tau^{2}}{2} \\
=y[(\mathrm{k}+\rho) \mathrm{T}]+\sigma_{\mathrm{k}}(\rho, \tau)+\delta_{\mathrm{k}}(\rho, \tau) \beta
\end{aligned}
$$

For $\tau \in(0,(1-\rho) \mathrm{T})$ with $\alpha=0$ for a first-order approximation and $\alpha=1$ for a second-order one, where:

$$
\begin{aligned}
& \sigma_{k}(\rho, \tau)=c^{T}\left\{\left(I+\alpha A \frac{\tau}{2}\right)\left[A \Phi(\rho T) x_{k}+(A \Gamma(\rho T)+b) u_{k}\right]\right\} \\
& \delta_{k}(\rho, \tau)=T^{-1} c^{T}\left\{\left(I+\alpha A \frac{\tau}{2}\right) \tau\left[A \Gamma^{\prime}(\rho T)+b \rho T\right]+\alpha \frac{b \tau^{2}}{2}\right\} \tilde{u}_{k-1}
\end{aligned}
$$

Choice of $\boldsymbol{\beta}$ for Minimal Output Deviations In-between Samples: Consider the inter-sample-output deviation performance function:

$$
\mathrm{J}_{\mathrm{k}}(\rho, \beta)=\int_{\rho \mathrm{T}}^{\mathrm{T}}(\mathrm{y}[(\mathrm{k}+\rho) \mathrm{T}+\tau]-\mathrm{y}[(\mathrm{k}+\rho) \mathrm{T}])^{2} \mathrm{~d} \tau \cong \int_{\rho \mathrm{T}}^{\mathrm{T}}\left(\sigma_{\mathrm{k}}(\rho, \tau)+\beta \delta_{\mathrm{k}}(\rho, \tau)\right)^{2} \mathrm{~d} \tau
$$

Which reaches a minimum from direct evaluation of the derivative with respect to $\beta$ at:

$\beta=\bar{\beta}_{\alpha k}=-\frac{\sigma_{k}(\rho, T)}{\delta_{k}(\rho, T)}$

Where:

$$
\begin{aligned}
& \sigma_{k}(\rho, T)=c^{\mathrm{T}} \mathrm{T}\left\{\left[\left(1-\rho^{2}\right) \frac{\mathrm{T}}{2}+\frac{\alpha\left(1-\rho^{3}\right) \mathrm{T}^{2} \mathrm{~A}}{6}\right] \mathrm{A} \Phi(\rho \mathrm{T}) \mathrm{x}_{\mathrm{k}}+(\mathrm{A} \Gamma(\rho \mathrm{T})+\mathrm{b})(1-\rho)\right\} \mathrm{u}_{\mathrm{k}} \\
& \delta_{k}(\rho, \mathrm{T})=\frac{\mathrm{c}^{\mathrm{T}}}{2}\left\{\left[\left(1-\rho^{2}\right) \mathrm{T}+\frac{\alpha\left(1-\rho^{3}\right) \mathrm{T}^{2} \mathrm{~A}}{6}\right]\left(\mathrm{A} \Gamma^{\prime}(\rho \mathrm{T})+\mathrm{b} \rho \mathrm{T}\right)+\frac{\alpha \mathrm{b}\left(1-\rho^{3}\right) \mathrm{T}^{2}}{3}\right\} \tilde{\mathrm{u}}_{\mathrm{k}-1}
\end{aligned}
$$

Since $J_{k}(\rho, \beta)$ is a convex function of $\beta$ for any real $\rho \in(0,1)$, a constrained minimization of $J_{k}(\rho, \beta)$ on $\left[\beta_{1}, \beta_{2}\right]$ is performed by an adjustable sample-dependent correcting gain as follows:

$\beta_{k}=\left\{\begin{array}{ccc}\beta_{2} & \text { if } & \bar{\beta}_{\alpha k} \geq \beta_{2 k} \\ \beta_{1} & \text { if } & \bar{\beta}_{\alpha k} \leq \beta_{1 k} \\ \bar{\beta} & \text { if } & \bar{\beta}_{\alpha k} \in\left[\beta_{1}, \beta_{2}\right]\end{array}\right.$

Example: Consider the linear and time-invariant continuous closed-loop system:

$\dot{\mathrm{x}}_{1}(\mathrm{t})=\mathrm{x}_{2}(\mathrm{t}) ; \dot{\mathrm{x}}_{2}(\mathrm{t})=-\mathrm{x}_{2}(\mathrm{t})+\left(1(\mathrm{t})-\mathrm{x}_{1}(\mathrm{t})\right) ; \mathrm{y}(\mathrm{t})=\mathrm{x}_{1}(\mathrm{t})$

Which corresponds to a field-flowered plant of transfer function $1 / \mathrm{s}(\mathrm{s}+1)$ under unity negative feedback and external unity step reference input. Such a continuous system is discretized via a FROH placed at its plant input with a sampling period $\mathrm{T}=0.1$ secs. The optimal fractional gain according to a second-order approximation of the loss performance (9) with $\beta=0$; i.e. $\beta=1$ in (10), is $\beta=-0.4$. The two better averaged positive and negative values of $\beta$ over 200 samples leading to the two minimal squared inter-sample tracking error (9) are reached at $\beta=$ 0.51 and $\beta=-0.40$, respectively, taken as the better averages of the results obtained from (12) over 200 samples. The corresponding tracking errors are displayed in Fig. 1 and 2. 
American J. Applied Sci., 2 (4): 771-773, 2005

Table 1: Relevant Portion of the Transient Output for Significant Values of $\beta$

\begin{tabular}{|c|c|c|c|c|c|c|c|c|c|c|}
\hline $\begin{array}{l}\beta=0(\mathrm{ZOH} \\
\text { Loss }(9)=\end{array}$ & & & & $\begin{array}{c}0.46 \\
25.9165\end{array}$ & & & & $\begin{array}{r}1(\mathrm{FC} \\
94.53\end{array}$ & & 180.282 \\
\hline$\beta=-0.25$ & -0.26 & -0.28 & -0.30 & -0.33 & -0.34 & -0.38 & -0.40 & -0.5 & -1 & \\
\hline $\operatorname{Loss}(9)=$ & 26. 28 & 26.96 & 26.962 & 1.92339 & 14.076 & 7.7730 & 2.0779 & 1.92 & 5.99167 & 3.32083 \\
\hline
\end{tabular}

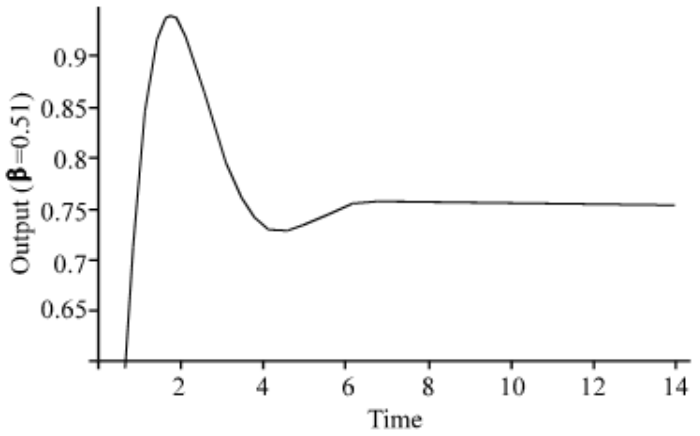

Fig. 1: Output for Correcting Gain 0.51

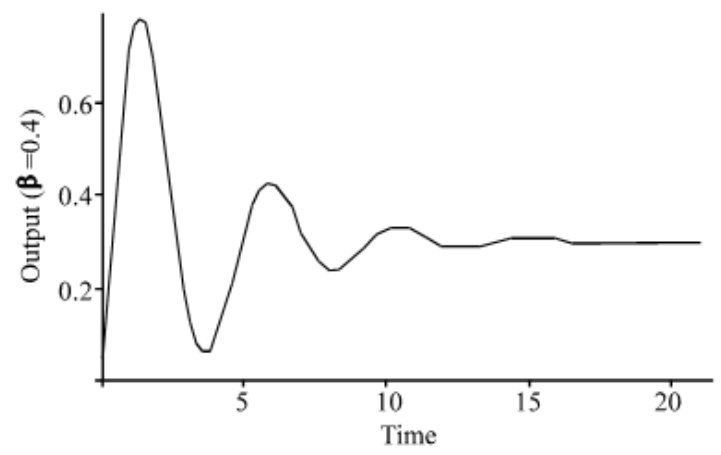

Fig. 2: Output for Correcting Gain -0.4

The values of the first exact expression (9) over 10000 samples are 305.559 for $\beta=-0.4$ and 409.191 for $\beta=$ 0.51 , respectively. A set of exact losses (9) over the first 100 samples describing a relevant portion of the transient output for significant values of $\beta$ is displayed in the Table 1.

Comments: It might be noticed that, in general, neither the widely used zero-order hold or the first-order hold are the best options for reconstruction of the output inbetween sampling instants closely to its sampled values. Note also that such a behavior is highly dependent on the chosen correcting fractional hold gain. A third point is that the correcting gain might be theoretically be designed as being sampled dependent if the basic suboptimization rule (12) is implemented but it can also be chosen constant through performing an averaging procedure as given in the example. A final interesting point is that the way of designing $\beta$ is dependent on the property one desires to enhance. For instance, negative values are good to achieve stable discrete zeros as the sampling period tends to zero if the system is of relative degree at most two. Negative values of the correcting gain (including the $\mathrm{ZOH} \beta=0$ ) may be appropriate for stabilization of discrete zeros if the sum of all continuous zeros becomes to be negative $\mathrm{e}^{[3,4]}$.

\section{REFERENCES}

1. De La Sen, M., 1988. A method for general design of positive real functions. IEEE Trans., pp: 764769, CASI-45, 7: 764-769.

2. De La Sen, M., 1999. Relationships between positive realness of continuous transfer functions and their digital counterparts. Elec. Lett., 35: 1298-1299.

3. Liang, S., M. Ishitobi and Q. Zhu, 2003. Improvement of stability of zeros in discrete multivariable systems using fractional-order hold. Int. J. Control, 76: 1699-1711.

4. Liang, S. and M. Ishitobi, 2004. The stability properties of the zeros of sampled models for timedelay systems in fractional order hold case. Dynam. Cont. Dis. Ser. B, 11: 299-312. 